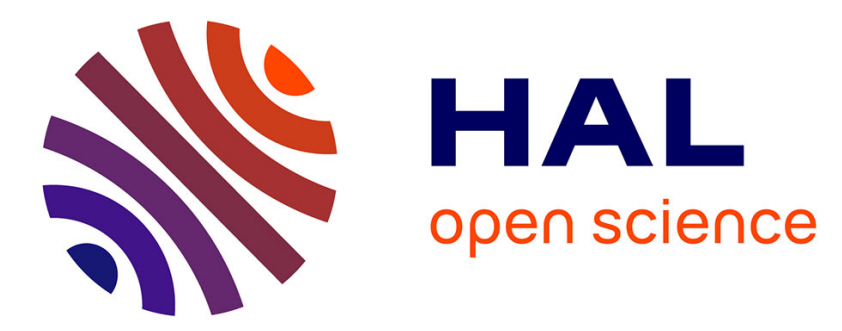

\title{
Magnetic-field-induced avalanches in magnetization and magnetoresistance of La0.9Ce0.1Fe12B 6 compound
}

\author{
L. V. B. Diop, O. Isnard
}

\section{To cite this version:}

L. V. B. Diop, O. Isnard. Magnetic-field-induced avalanches in magnetization and magnetoresistance of La0.9Ce0.1Fe12B6 compound. The European Physical Journal Plus, 2021, 136 (8), pp.840. 10.1140/epjp/s13360-021-01842-w . hal-03325814

\section{HAL Id: hal-03325814 \\ https://hal.science/hal-03325814}

Submitted on 25 Aug 2021

HAL is a multi-disciplinary open access archive for the deposit and dissemination of scientific research documents, whether they are published or not. The documents may come from teaching and research institutions in France or abroad, or from public or private research centers.
L'archive ouverte pluridisciplinaire HAL, est destinée au dépôt et à la diffusion de documents scientifiques de niveau recherche, publiés ou non, émanant des établissements d'enseignement et de recherche français ou étrangers, des laboratoires publics ou privés. 


\title{
Magnetic-field-induced avalanches in magnetization and magnetoresistance of
} La0.9 Ce.1Fe12 $\mathrm{B}_{6}$ compound

\author{
L.V.B. Diop ${ }^{1 \dagger}$ and O. Isnard ${ }^{2}$ \\ ${ }^{1}$ Université de Lorraine, CNRS, IJL, F-54000 Nancy, France \\ ${ }^{2}$ Université Grenoble Alpes, Institut NEEL CNRS, 25 rue des martyrs, F-38042 Grenoble, France
}

Competition between paramagnetic (PM), antiferromagnetic (AFM) and ferromagnetic (FM) phases in $\mathrm{La}_{0 .} \mathrm{Ce}_{0.1} \mathrm{Fe}_{12} \mathrm{~B}_{6}$ itinerant-electron metamagnetic system is studied by a combination of magnetic, electrical resistivity and magnetoresistance measurements. $\mathrm{La}_{0.9} \mathrm{Ce}_{0.1} \mathrm{Fe}_{12} \mathrm{~B}_{6}$ is an antiferromagnet below the Néel temperature (35 K) and presents two consecutive magnetic transitions AFM-FM and FM-PM during warming under certain applied magnetic field values. We further demonstrate that both AFM and PM phases may be converted into the FM phase irreversibly and reversibly via a first-order metamagnetic transition accompanied by a large magnetic field hysteresis. At very low temperatures, the magnetic-field driven AFM-FM metamagnetic transformation is discontinuous and proceeds by multiple abrupt steps in magnetization and magnetoresistance. In addition, an extraordinarily large negative magnetoresistance (MR) effect of $-77 \%$ is observed. The consistency found in the magnetotransport and magnetization data suggests strong correlations between charge and spin degrees of freedom in $\mathrm{La}_{0 .} \mathrm{Ce}_{0.1} \mathrm{Fe}_{12} \mathrm{~B}_{6}$ system.

\section{INTRODUCTION}

Itinerant electron metamagnetism (IEM) constitutes a crucial physical property, depicting magnetic behaviors of intermetallic systems, manifesting potentially important functionalities like large magnetostrictive, magnetoresistive, and magnetocaloric effects [1-4]. Giant magnetocaloric effects discovered in the itinerant-electron metamagnetic compound $\mathrm{La}(\mathrm{Fe}, \mathrm{Si})_{13}$ [1] have stimulated the development of many studies worldwide on both the fundamental research side as well as the technological applications viewpoint (magnetic refrigeration).

One intriguing example of IEM is the ternary system $\mathrm{LaFe}_{12} \mathrm{~B}_{6}$ which occupies a special place among rare-earth iron-rich alloys. $\mathrm{LaFe}_{12} \mathrm{~B}_{6}$ presents many striking features and exotic magnetic behavior. Discontinuous and unconventional avalanche-like metamagnetic phase transitions were recently found in $\mathrm{LaFe}_{12} \mathrm{~B}_{6}$ [5-8]. This peculiar and unusual multistep

\footnotetext{
${ }^{+}$leopold.diop@univ-lorraine.fr
} 
magnetization process is characterized by extremely sharp jumps followed by plateaus. An amplitude-modulated magnetic structure defined by a propagation vector $\mathbf{k}=(1 / 4,1 / 4,1 / 4)$ and especially weak Fe magnetic moment of about $0.43 \mu_{\mathrm{B}}$ were revealed by neutron powder diffraction for the antiferromagnetic ground state [5]. $\mathrm{LaFe}_{12} \mathrm{~B}_{6}$ exhibits extraordinary low Néel temperature $T_{\mathrm{N}}=36 \mathrm{~K}$ for an Fe-rich intermetallic phase, a critical point in the complex magnetic phase diagram [5], both inverse and normal magnetocaloric phenomenon [9], huge spontaneous magnetization steps [7], and large magnetovolume effects [10]. These singular characteristics not only tendered the development of investigations under extreme conditions and theoretical models for a deeper understanding of the interesting physics underlying the striking behavior of this intermetallic compound [10-14], but also highlighted the potential interest of $\mathrm{LaFe}_{12} \mathrm{~B}_{6}$ in future low-temperature energy technologies. $\mathrm{LaFe}_{12} \mathrm{~B}_{6}$ constitutes an exceptional playground for materials physics due to the extreme sensitivity of its intrinsic physical properties to hydrostatic pressure [10] and chemical substitution that yields the effect of "chemical pressure" [8].

Among the $R T_{12} \mathrm{~B}_{6}$ intermetallics (where $R$ is a rare-earth atom and $T$ stands for a $3 d$ transition metal element $\mathrm{Co}$ or $\mathrm{Fe}$ ) the $R \mathrm{Co}_{12} \mathrm{~B}_{6}$ phases are stable along the entire rare-earth series (but not reported yet for europium and promethium), whereas $\mathrm{LaFe}_{12} \mathrm{~B}_{6}$ is the sole stable Fe-based alloy of the 1:12:6 ternary system [13-15]. Even though $\mathrm{NdFe}_{12} \mathrm{~B}_{6}$ phase was the first Fe-based member of the $R T_{12} \mathrm{~B}_{6}$ family to be identified, it is not stable [16]. At ambient conditions, the $R T_{12} \mathrm{~B}_{6}$ series of compounds adopts the rhombohedral $\mathrm{SrNi}_{12} \mathrm{~B}_{6}$-type crystallographic structure with $R \overline{3} m$ space group [17-19]. In such atomic arrangement, there are two inequivalent positions for $T$ atoms (18g and $18 h$ ). The $R$ and $\mathrm{B}$ atoms reside on the $3 a$ and $18 h$ Wyckoff sites, respectively. $\mathrm{LaFe}_{12} \mathrm{~B}_{6}$ compound is unique within the $R T_{12} \mathrm{~B}_{6}$ family exhibiting an antiferromagnetic ground state with an ordering temperature much smaller than the Curie temperature of the Co-based $R \mathrm{Co}_{12} \mathrm{~B}_{6}$ ferro- $(R=\mathrm{Y}, \mathrm{La}-\mathrm{Sm})$ or ferri- $(R=\mathrm{Gd}-\mathrm{Tm})$ magnets $\left(T_{\mathrm{C}}=134-162 \mathrm{~K}\right)$ [15]. The Néel temperature of $\mathrm{LaFe}_{12} \mathrm{~B}_{6}$ is an order of magnitude smaller when compared to the magnetic transition temperature of any rare-earth iron-rich binary alloy. Extraordinary magnetotransport properties have been most recently reported in $R \mathrm{Co}_{12} \mathrm{~B}_{6}$ intermetallics with $R=\mathrm{Y}$, Gd and Ho [20].

To the best of our knowledge, no studies have been reported on the magnetotransport properties of a Fe-based member of the $R T_{12} \mathrm{~B}_{6}$ family. The present work aims to thoroughly investigate the magnetic, electronic transport and magnetotransport properties of $\mathrm{La}_{0.9} \mathrm{Ce}_{0.1} \mathrm{Fe}_{12} \mathrm{~B}_{6}$ intermetallic compound by means of magnetization, electrical resistivity and magnetoresistance measurements. 


\section{EXPERIMENTS}

Details on the preparation and structural studies of the $\mathrm{La}_{0.9} \mathrm{Ce}_{0.1} \mathrm{Fe}_{12} \mathrm{~B}_{6}$ polycrystalline sample can be found in Ref. 8. Magnetization measurements were performed on a powder sample with an extraction-type magnetometer. Temperature and magnetic field dependencies of the magnetization were measured in applied fields of up to $10.5 \mathrm{~T}$. Zero-field cooled warming (ZFCW), field cooled cooling (FCC) and field cooled warming (FCW) measuring protocols were employed for thermomagnetic measurements. The ferromagnetic contribution of the minor $\mathrm{Fe}_{2} \mathrm{~B}$ secondary phase was subtracted from the magnetization data. The magnetometer is described in Ref. 21. For the electrical resistivity and magnetoresistance characterizations, the sample was cut in parallelepiped form using diamond saw and subsequently smooth and flat surfaces were prepared by polishing. The contacts on the sample' surface were made by fixing thin platinum wires using silver paste. The measurements were carried out using the conventional four-point contact method at a constant $d c$ current of $10 \mathrm{~mA}$ over the temperature range between 2.5 and $125 \mathrm{~K}$ in a superconducting magnet providing a maximum field of $8 \mathrm{~T}$. The magnetic field was applied perpendicular to the current orientation $(H \perp i)$. In order to get rid of possible thermals, the $d c$ electrical current was applied in opposite polarities at each measurement point.

\section{RESULTS AND DISCUSSION}

(a)

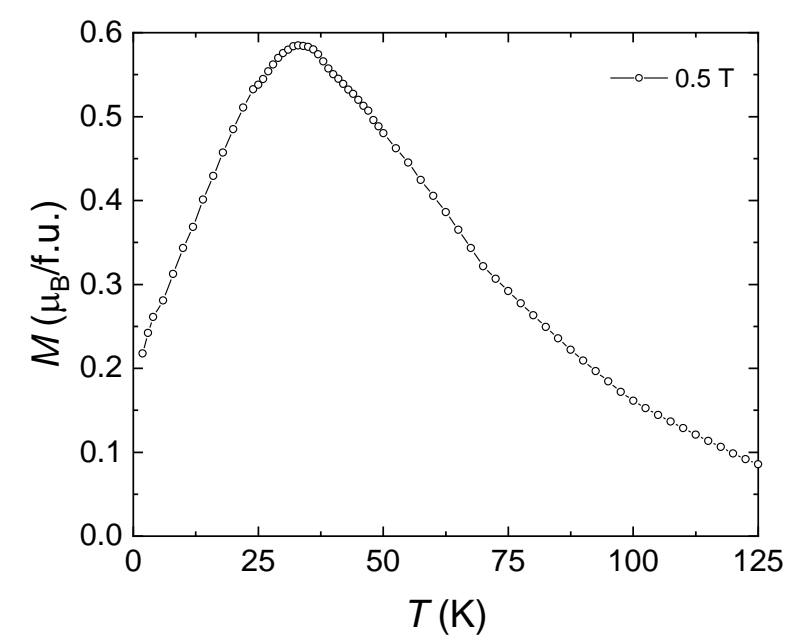

(b)

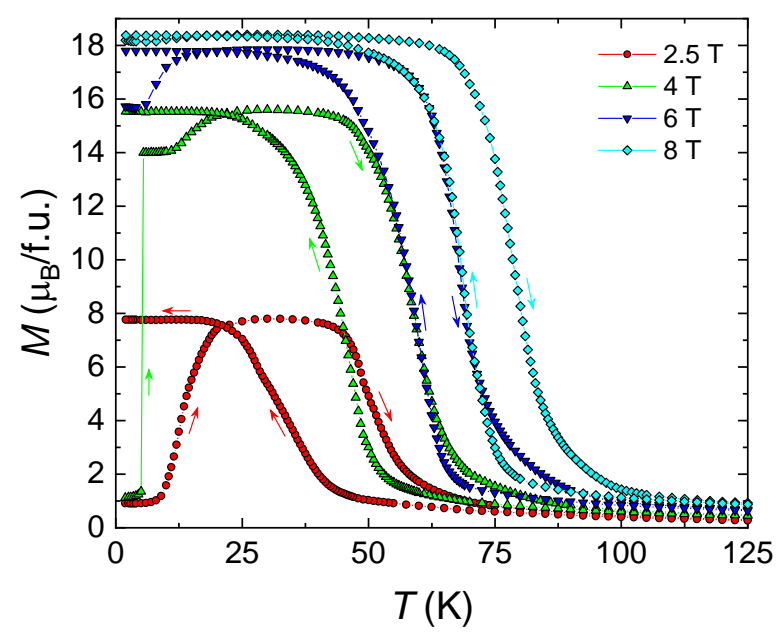

Figure 1. Temperature dependence of the magnetization of $\mathrm{La}_{0.9} \mathrm{Ce}_{0.1} \mathrm{Fe}_{12} \mathrm{~B}_{6}$ : (a) magnetization measured using ZFCW protocol in $0.5 \mathrm{~T}$ applied magnetic field; (b) magnetization measured in applied magnetic fields of 2.5, 4, 6 and $8 \mathrm{~T}$ (Both ZFCW and FCC data are marked by the same symbols. The arrows indicate the direction of the temperature change). 
The ZFCW and FCC temperature-dependent magnetization data, $M(T)$, recorded under various external magnetic fields are plotted in figure 1 . The $0.5 \mathrm{~T}$ thermomagnetic curve (figure 1a) exhibits a small peak around $35 \mathrm{~K}$ corresponding to the Néel temperature of the secondorder AFM-PM transition. In a $8 \mathrm{~T}$ applied magnetic field and low temperatures (figure 1b), La0.9 $\mathrm{Ce}_{0.1} \mathrm{Fe}_{12} \mathrm{~B}_{6}$ is in the fully $\mathrm{FM}$ polarized state, and thus the isofield curve shows the transition from a FM state to a PM phase. The $M(T)$ curve in the presence of $6 \mathrm{~T}$ applied field indicates that a large amount, $\sim 85 \%$, of the sample volume is converted into FM state at $2 \mathrm{~K}$, and the remaining proportion $\sim 15 \%$ is in the initial AFM phase. As can be clearly seen from figure $1 \mathrm{~b}$, the $2.5 \mathrm{~T}$ ZFCW thermomagnetic curve displays a bell-like anomaly reflecting the existence of two successive transformations: AFM-FM transformation at low temperatures and FM-PM phase transition at high temperatures. When cooled in a $2.5 \mathrm{~T}$ magnetic field, $\mathrm{La}_{0.9} \mathrm{Ce}_{0.1} \mathrm{Fe}_{12} \mathrm{~B}_{6}$ gets converted into a partially FM phase. Of particular interest is the $4 \mathrm{~T}$ isofield curve where the magnetization presents an abrupt step when temperature is increased by $0.5 \mathrm{~K}$. One can remark a strong temperature hysteresis across the FM-PM transition, which confirms the first-order character of the phase transformation. The Curie temperature, $T_{\mathrm{C}}$, has been derived from the thermomagnetic curves such as the ones shown in figure $1 \mathrm{~b} . T_{\mathrm{C}}$ was determined as the abscissa of the crossing point of the high-temperature part of the extended baseline and the linear portion of the isofield magnetization curves. $T_{\mathrm{C}}$ of $\mathrm{La} 0 .{ }_{9} \mathrm{Ce}_{0.1} \mathrm{Fe}_{12} \mathrm{~B}_{6}$ is plotted in figure 2 as a function of the applied external magnetic field. The application of magnetic field leads to a nearly linear increase of $T_{\mathrm{C}}$ at a rate of 5.6 and $4.9 \mathrm{~K} / \mathrm{T}$ upon cooling and warming, respectively. This demonstrates that the magnetic field enhances the FM state.

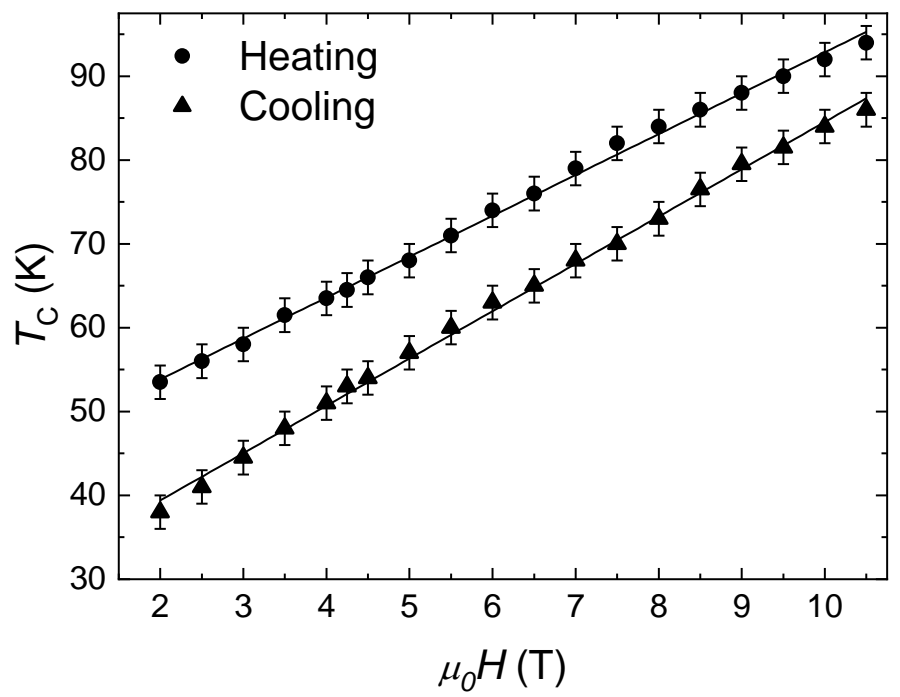

Figure 2. Magnetic field dependence of the Curie temperature, $T_{\mathrm{C}}$, for $\mathrm{La}_{0.9} \mathrm{Ce}_{0.1} \mathrm{Fe}_{12} \mathrm{~B}_{6}$ as derived from thermomagnetic measurements. 


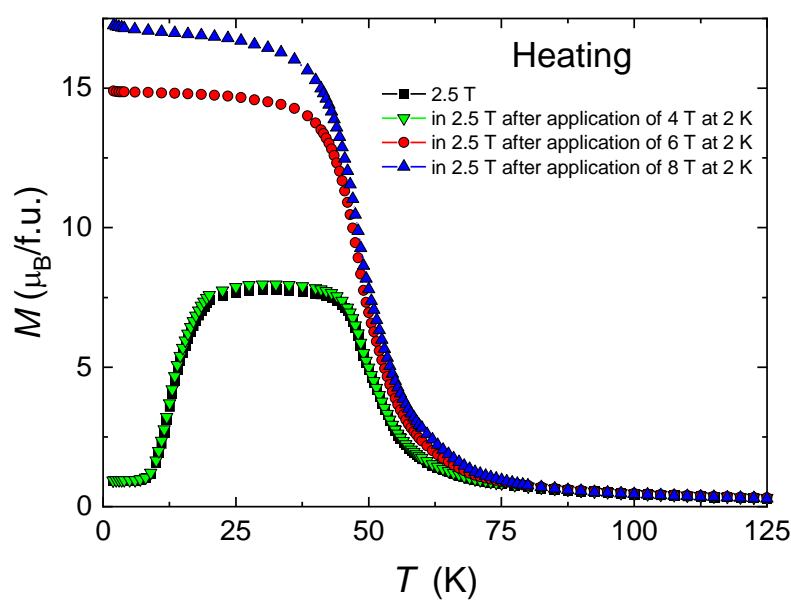

Figure 3. Thermomagnetic curves of $\mathrm{La}_{0.9} \mathrm{Ce}_{0.1} \mathrm{Fe}_{12} \mathrm{~B}_{6}$ measured on heating under various indicated conditions.

Figure 3 shows the temperature dependence of the magnetization of the zero-magneticfield cooled $\mathrm{La}_{0.9} \mathrm{Ce}_{0.1} \mathrm{Fe}_{12} \mathrm{~B}_{6}$ alloy recorded upon heating under various conditions. These thermomagnetic data were collected in a $2.5 \mathrm{~T}$ external field after the sample was premagnetized at $2 \mathrm{~K}$ by the application of magnetic fields $\mu_{0} H=4,6$ and $8 \mathrm{~T}$. When premagnetized at $2 \mathrm{~K}$ in a $6 \mathrm{~T}$ magnetic field, a big fraction, $\sim 81 \%$, of the sample volume is transformed into a FM state, and $\sim 19 \%$ remains in the AFM state. Note that the fraction of induced-FM phase depends on the magnitude of applied magnetic field, which favors the FM state over the AFM state. According to figure 3, the magnetic ordering temperature of the PMFM phase transformation is hardly changed, whether $\mathrm{La}_{0.9} \mathrm{Ce}_{0.1} \mathrm{Fe}_{12} \mathrm{~B}_{6}$ at $2 \mathrm{~K}$ was originally in the antiferromagnetic ground state or at first converted to the ferromagnetic state. Conversely, the magnetic history of the sample influences strongly the value of maximal magnetization at $\sim 32 \mathrm{~K}$ (center of the bell-shaped anomaly figure 3): the total magnetization of $\mathrm{La} 0 .{ }_{9} \mathrm{Ce}_{0.1} \mathrm{Fe}_{12} \mathrm{~B}_{6}$ which initially was in the forced FM state is much larger than that of the same $\mathrm{La}_{0 .} \mathrm{Ce}_{0.1} \mathrm{Fe}_{12} \mathrm{~B}_{6}$ system, which originally was in the AFM ground state. These results reveal that the temperature alone cannot fully convert the AFM phase into a FM state during warming in a $2.5 \mathrm{~T}$ applied field.

Figure 4 represents the temperature dependence of the magnetization in $3 \mathrm{~T}$ under ZFCW, FCC, and FCW conditions. In the ZFCW leg, the tremendous change in magnetization by $630 \%$, when temperature is increased by $10 \mathrm{~K}$, is associated with the instantaneous formation of the FM phase. The magnetic state of the thermally demagnetized $\mathrm{La}_{0.9} \mathrm{Ce}_{0.1} \mathrm{Fe}_{12} \mathrm{~B}_{6}$ evolves from the AFM order to PM via a partially FM phase upon heating. During the FCC cooling process, the magnetization grows rapidly as the temperature is lowered and then saturates, revealing that the magnetic phase transformation in $\mathrm{La}_{0 .} \mathrm{Ce}_{0.1} \mathrm{Fe}_{12} \mathrm{~B}_{6}$ depends on the direction 
of the temperature variation. Additionally, the maximum value of magnetization is larger for the ZFCW branch than for the FCC and FCW measuring protocols. This feature is rather uncommon for conventional ferromagnetic systems in an applied magnetic field as large as 3 T. Figure 4 shows a marked divergence between ZFCW, FCC and FCW data. Another observation in these thermomagnetic curves is the large difference in magnetization at low temperatures between FCC and FCW modes. At this stage, it is noteworthy that the FCC thermomagnetic curve was measured upon slow cooling, by contrast, the FCW data were collected after rapid cooling. Consequently, the cooling rate under applied field influences the fraction of the sample converted into the FM phase at $2 \mathrm{~K}$. According to figure 4 , the Curie temperature of the FM-PM transition during heating is not affected, whether $\mathrm{La}_{0.9} \mathrm{Ce}_{0.1} \mathrm{Fe}_{12} \mathrm{~B}_{6}$ at $2 \mathrm{~K}$ was in the AFM ground state or partially converted to the FM state.

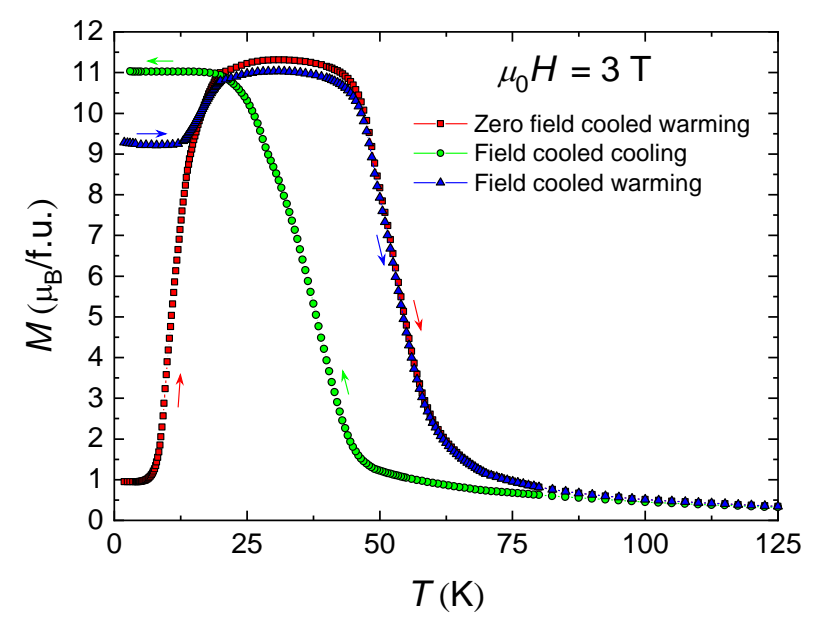

Figure 4. Temperature dependence of the magnetization of $\mathrm{La}_{0.9} \mathrm{Ce}_{0.1} \mathrm{Fe}_{12} \mathrm{~B}_{6}$ in magnetic field of $2.5 \mathrm{~T}$ using ZFCW, FCC and FCW modes. The arrows indicate the direction of the temperature change.

Magnetization isotherms, $M(H)$, of the thermally demagnetized $\mathrm{La}_{0.9} \mathrm{Ce}_{0.1} \mathrm{Fe}_{12} \mathrm{~B}_{6}$ are shown in figure 5 . At $3 \mathrm{~K}$, the first magnetization curve (virgin curve) displays two abrupt jumps followed by plateaus; generating a staircase-like metamagnetic process. These avalanche-like transitions result from conversion of a proportion of the specimen volume from the AFM state into the FM state. Both AFM and FM phases coexist throughout the first magnetization plateau, hence forming a magnetically inhomogeneous state (mixed phase AFM+FM). The saturation magnetization of the fully FM polarized phase is estimated to be $18.20 \mu_{\mathrm{B}} /$ f.u. at $3 \mathrm{~K}$. The demagnetization loop exhibits no steps neither transitions and shows a standard ferromagnetic behavior, indicating that the entire sample stays in the magnetic-field induced FM state after the applied field is reduced to zero. The field-driven AFM-FM transition is totally irreversible at this temperature. 


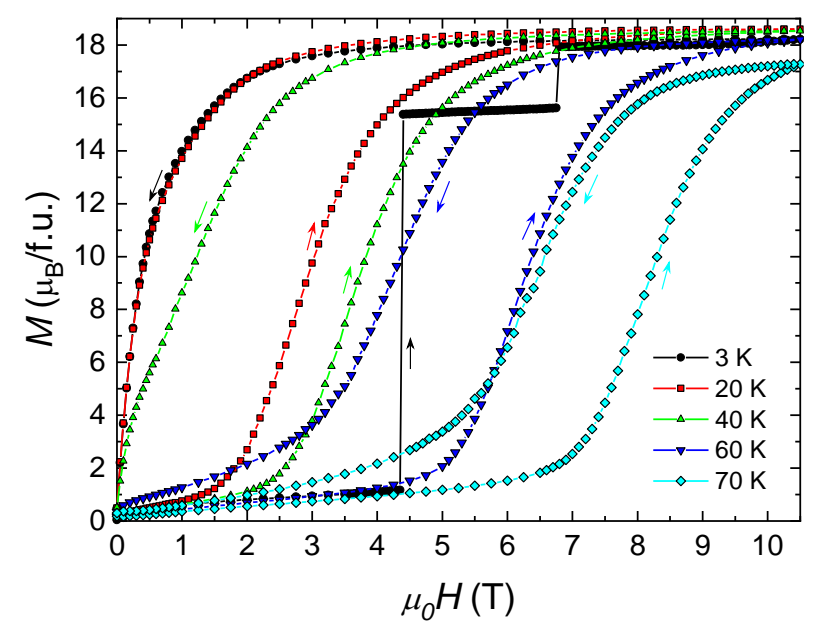

Figure 5. Magnetization isotherms of $\mathrm{La}_{0.9} \mathrm{Ce}_{0.1} \mathrm{Fe}_{12} \mathrm{~B}_{6}$ measured between 3 and $70 \mathrm{~K}$.

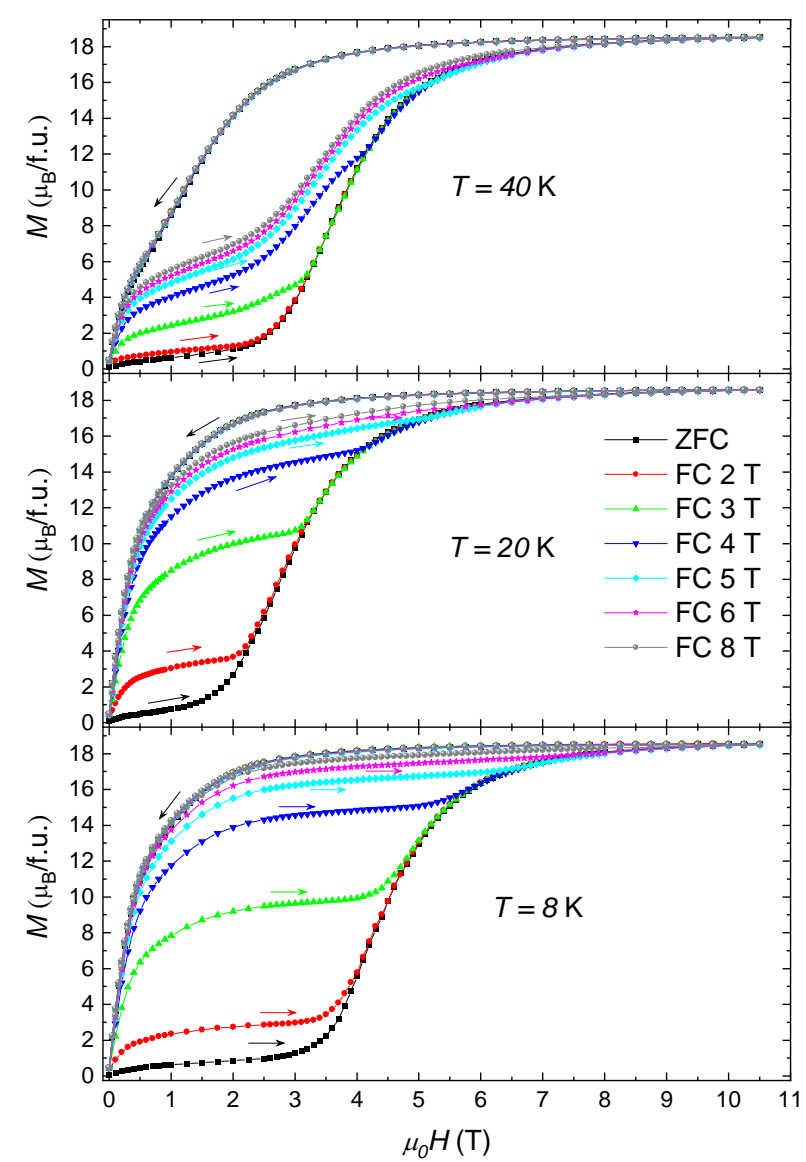

Figure 6. Magnetization isotherms of $\mathrm{La}_{0.9} \mathrm{Ce}_{0.1} \mathrm{Fe}_{12} \mathrm{~B}_{6}$ at some selected temperatures of $8 \mathrm{~K}$ (bottom panel) $20 \mathrm{~K}$ (middle panel) and $40 \mathrm{~K}$ (top panel) taken after cooling the sample in different magnetic fields as indicated.

The sharpness of the step-like metamagnetic transitions is reduced upon heating and disappearing at $8 \mathrm{~K}$ where the magnetization process becomes continuous. In the itinerantelectron compound $\mathrm{La}_{0.9} \mathrm{Ce}_{0.1} \mathrm{Fe}_{12} \mathrm{~B}_{6}$ the metamagnetic transition extends over a wide temperature span, below and well beyond $T_{\mathrm{N}}$, proceeding above $8 \mathrm{~K}$ via a progressive 
conversion of the AFM and PM states into FM domains upon increasing external magnetic field. A large magnetic hysteresis accompanies the first-order AFM-FM and PM-FM phase transitions. For instance, the width of the hysteresis amounts to $2.6 \mathrm{~T}$ at $40 \mathrm{~K}$. Considering the metastable character of the different magnetic states, we further investigated the effects of the field strength applied during cooling on the proportion of the AFM, FM, and PM phases and on the metamagnetic process. The results obtained at $8 \mathrm{~K}, 20 \mathrm{~K}$ and $40 \mathrm{~K}$ are depicted in figure 6 . One can observe the increase of the FM phase fraction to the detriment of the AFM or PM ones. The critical field of the metamagnetic transition is hardly changed during this field cooling procedure.

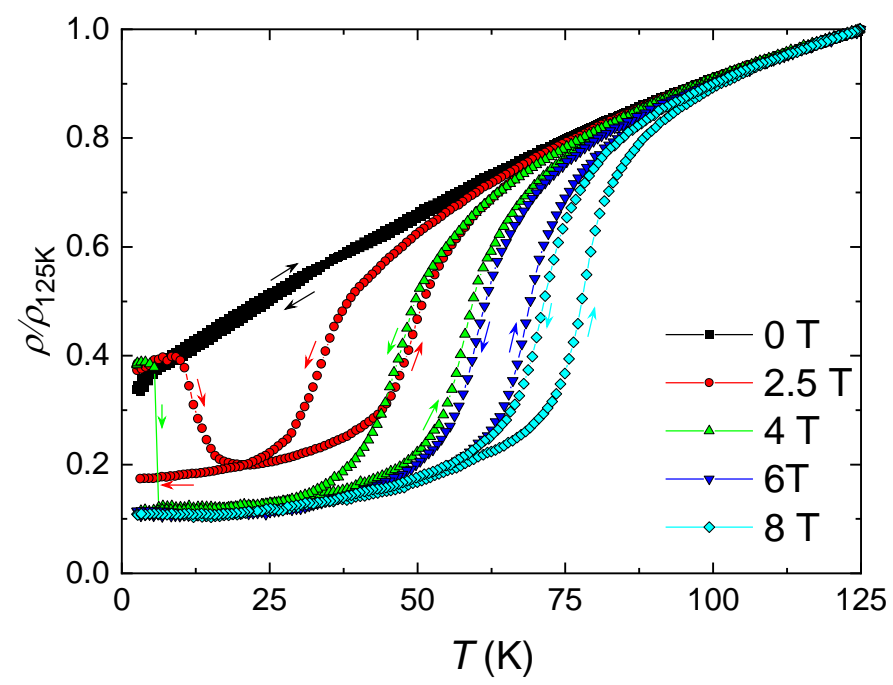

Figure 7. Temperature dependence of the relative electrical resistivity of $\mathrm{La}_{0.9} \mathrm{Ce}_{0.1} \mathrm{Fe}_{12} \mathrm{~B}_{6}$ on heating and cooling in various applied magnetic fields. Both ZFC and FC data are marked by the same symbols. The arrows indicate the direction of the temperature change.

The thermal dependence of the resistivity, $\rho(T)$, of $\mathrm{La}_{0.9} \mathrm{Ce}_{0.1} \mathrm{Fe}_{12} \mathrm{~B}_{6}$ in $\mathrm{ZFCW}$ and FCC measuring protocols under various magnetic fields, is reported in figure 7. At high temperatures, the electrical resistivity presents a strongly linear variation with respect to temperature; consistent with the metallic behavior. In the absence of applied field $\left(\mu_{0} H=0 \mathrm{~T}\right)$ both ZFCW and FCC $\rho(T)$ curves are nearly identical; no perceptible anomaly is observed at $T_{\mathrm{N}}$. The huge change in resistivity around $80 \mathrm{~K}(70 \mathrm{~K})$ during warming under $8 \mathrm{~T}(6 \mathrm{~T})$ ensues from magnetic transition from FM (low resistivity) to PM (high resistivity) phase. These values of the magnetic ordering temperature are in accord with those determined from the temperature dependence of the magnetization. In the vicinity of the Curie temperature, the increase of applied field yields the diminution of the resistivity due to decreased spin scattering by the magnetic field induced orientation of the local magnetic moments. The dramatic variation in the resistivity at $T_{\mathrm{C}}$ 
demonstrates a strong interaction of Fe magnetic moments with conduction electrons.

The electrical resistivity reported in figure 7 shows considerably different thermal behavior when recorded in $2.5 \mathrm{~T}$ magnetic field : on warming from the initial AFM ground state at $2.5 \mathrm{~K}$, the resistivity first decreases at the onset of the FM order and then grows at high temperatures, leading to basin-like feature. This peculiar thermal dependence is associated with the presence of both high-temperature FM-PM and low-temperature AFM-FM magnetic phase transformations. The $2.5 \mathrm{~T}$ isofield $\rho(T)$ curve presents a strong splitting between ZFCW and FCC modes and a large temperature hysteresis of about $14 \mathrm{~K}$ which highlights the first-order nature of the phase transition. Unexpectedly, the ZFCW 4 T $\rho(T)$ curve exhibits an abrupt jump at which the relative resistivity varies suddenly from 0.38 to 0.12 . This sharp discontinuity strikingly resembles that detected in the $4 \mathrm{~T}$ ZFCW thermomagnetic plot (see figure $1 \mathrm{~b}$ ) and points out the strong correlation between electronic transport and magnetism in $\mathrm{La}_{0.9} \mathrm{Ce}_{0.1} \mathrm{Fe}_{12} \mathrm{~B}_{6}$. The tremendous resistivity variation at the order-order AFM $\rightarrow$ FM transition can be ascribed to the difference in the strength of the scattering of the conduction electrons by the AFM and FM magnons and by the phonons. Our experimental results undoubtedly demonstrate that the scattering in the FM order is much smaller than that in the AFM magnetic structure.

To elucidate the resistivity change by the metamagnetic transition, magnetic field dependence of the electrical resistivity was measured at various fixed temperatures. Using these isothermal data, the magnetoresistance ratio MR was defined as $\left[\rho\left(\mu_{0} H, T\right)-\rho(0, T)\right] / \rho(0, T)$. Magnetoresistance isotherms of the zero field cooled sample are plotted in figures 8-9 at representative temperature intervals. At very low temperatures - figure 8 - during the first application of the magnetic field, the resistivity curve presents an abrupt stepwise variation in the same field range where a steep discontinuity was seen in the isothermal magnetization curves. This sharp and large change in the magnetoresistance is caused by the magnetic-fieldinduced first-order AFM-FM transition, with the AFM phase having a larger resistivity when compared with that of the forced FM state. The subsequent reverse branch shows no steps and the system remains in the induced-FM state. The initial value of the electrical resistivity is not recovered after reducing the applied magnetic field to zero; leading to the existence of a remanent (nonzero) magnetoresistance. This feature endorses the complete irreversibility of the AFM-FM metamagnetic transition in the very low temperature region. In order to return to the original AFM ground state and thus, the initial resistivity value, the sample should be heated above $T_{\mathrm{C}}$ and subsequently cooled in the absence of magnetic field. 


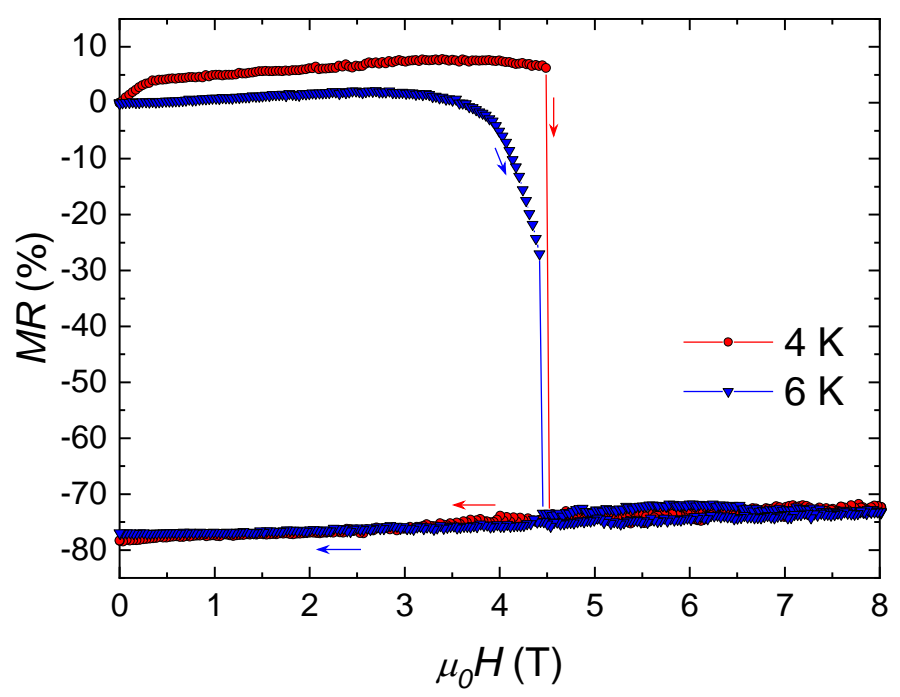

Figure 8 : Magnetic-field dependence of the magnetoresistance of $\mathrm{La}_{0.9} \mathrm{Ce}_{0.1} \mathrm{Fe}_{12} \mathrm{~B}_{6}$ at 4 and $6 \mathrm{~K}$.

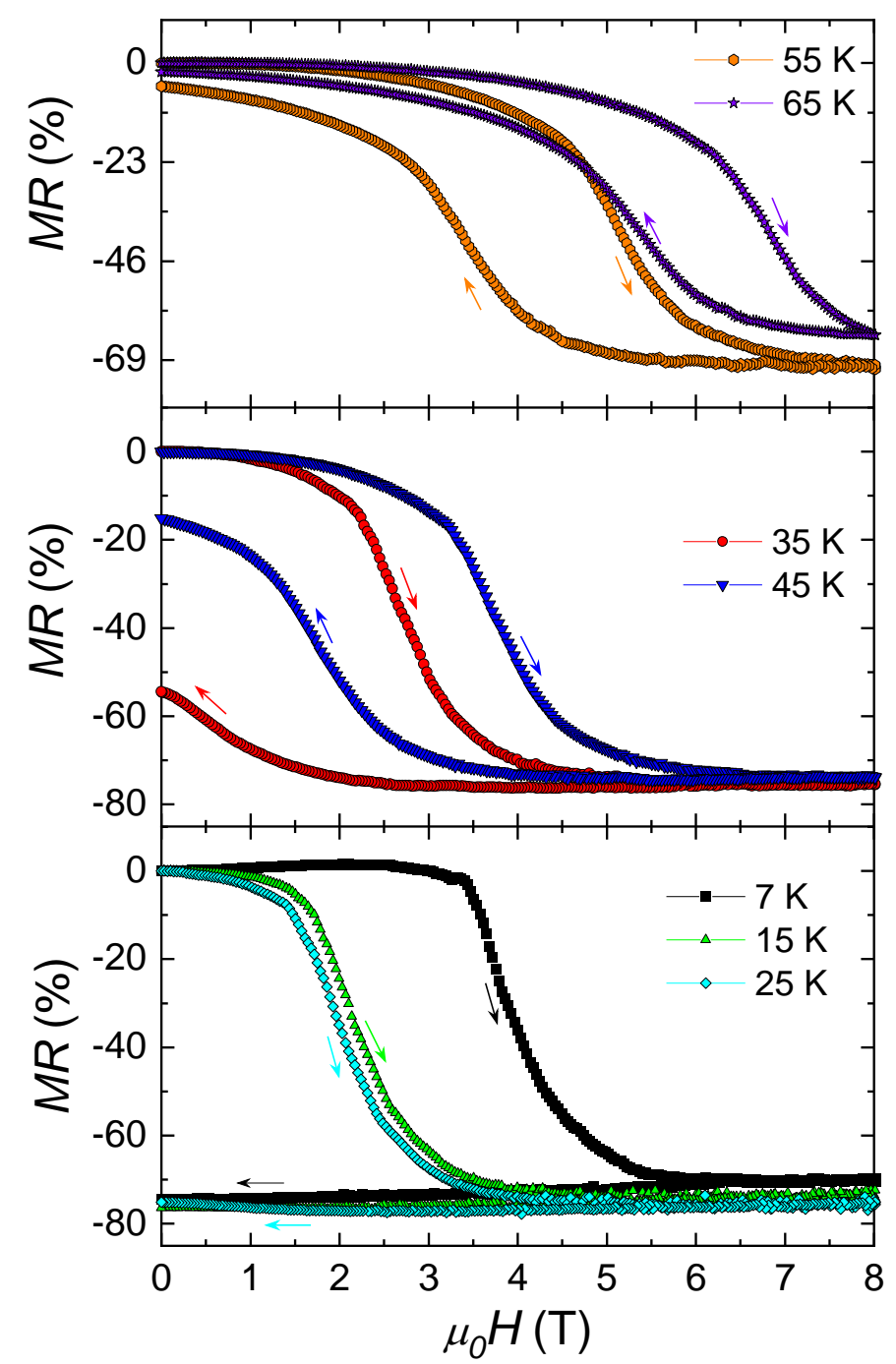

Figure 9. Magnetoresistance isotherms of $\mathrm{La}_{0.9} \mathrm{Ce}_{0.1} \mathrm{Fe}_{12} \mathrm{~B}_{6}$ for the temperature intervals 7 to $25 \mathrm{~K}$ (bottom panel), 35 to $45 \mathrm{~K}$ (middle panel) and 55 to $65 \mathrm{~K}$ (top panel). 
At temperatures ranging from 7 to $65 \mathrm{~K}$ (figure 9), the magnetoresistance isotherms display gradual changes across both AFM-FM and PM-FM magnetic phase transformations unlike the discontinuous process seen below $7 \mathrm{~K}$. The transitions are accompanied by a large magnetic field hysteresis which is one of the signatures of a first-order transformation. This hysteretic character and the irreversible/reversible nature depend on the temperature interval. Figure 9 illustrates three representative temperature ranges: between 7 and $25 \mathrm{~K}$ (bottom panel), from 35 to $45 \mathrm{~K}$ (middle panel) and $T \geq 55 \mathrm{~K}$ (top panel). Below $25 \mathrm{~K}$ in the AFM phase, the magnetoresistance ratio is small at low fields but remarkably diminishes as the alloy undergoes a magnetic transition to the FM phase giving rise to a giant negative MR effect. The magnetoresistance change due to the AFM-FM transformation is evaluated to be MR $=-77 \%$ at $25 \mathrm{~K}$. The resistivity remains essentially constant throughout the decreasing-field run, reflecting the irreversible nature of the transition at temperatures lower than $25 \mathrm{~K}$.

Above $25 \mathrm{~K}$ and below $55 \mathrm{~K}$, the isothermal magnetoresistance curve of the decreasingfield process deviates from the pure FM character and a metamagnetic-like transition occurs at lower field, producing a clear magnetic hysteresis between both field runs. In the temperature interval $25 \mathrm{~K}<T<55 \mathrm{~K}$, the field-driven AFM-FM and PM-FM transformations are partially reversible; a proportion of the sample volume recovers the initial AFM or PM state after the applied field is removed. In other words, both reversible and irreversible transitions coexist in the temperature region, and the percentage of the system, which endures the irreversible transformation, declines with increasing temperature. Beyond $55 \mathrm{~K}$, as manifested by isotherm at $65 \mathrm{~K}$, the initial value of the electrical resistivity is recovered when the magnetic field is brought back to zero. Simply meaning that the PM-FM transition becomes totally reversible, still with a hysteresis against the magnetic field scan.

By analogy to the magnetization measurements, we also examined the effects of the field strength applied during the cooling process on the electrical resistivity. For such investigations, the system was cooled in the presence of a magnetic field $\left(\mu_{0} H>0\right)$ from ambient temperature down to the measurement temperature. After stabilizing the desired temperature, the cooling field was removed, and afterward the electrical resistivity was recorded against external field up to $8 \mathrm{~T}$ and back to zero magnetic field. The resulting plots are reported in figure 10 for selected temperatures in the AFM and PM states. For both temperatures, $8 \mathrm{~K}$ and $40 \mathrm{~K}$, this field cooling procedure reduces the low-field resistivity owing to the rise of the FM phase proportion in the sample at the expense of the AFM and PM phases. At $8 \mathrm{~K}$, the critical field of the AFM-FM transition is not affected upon field cooling, however, the thermomagnetic history of the sample influences the transition field of the PM-FM transition at 


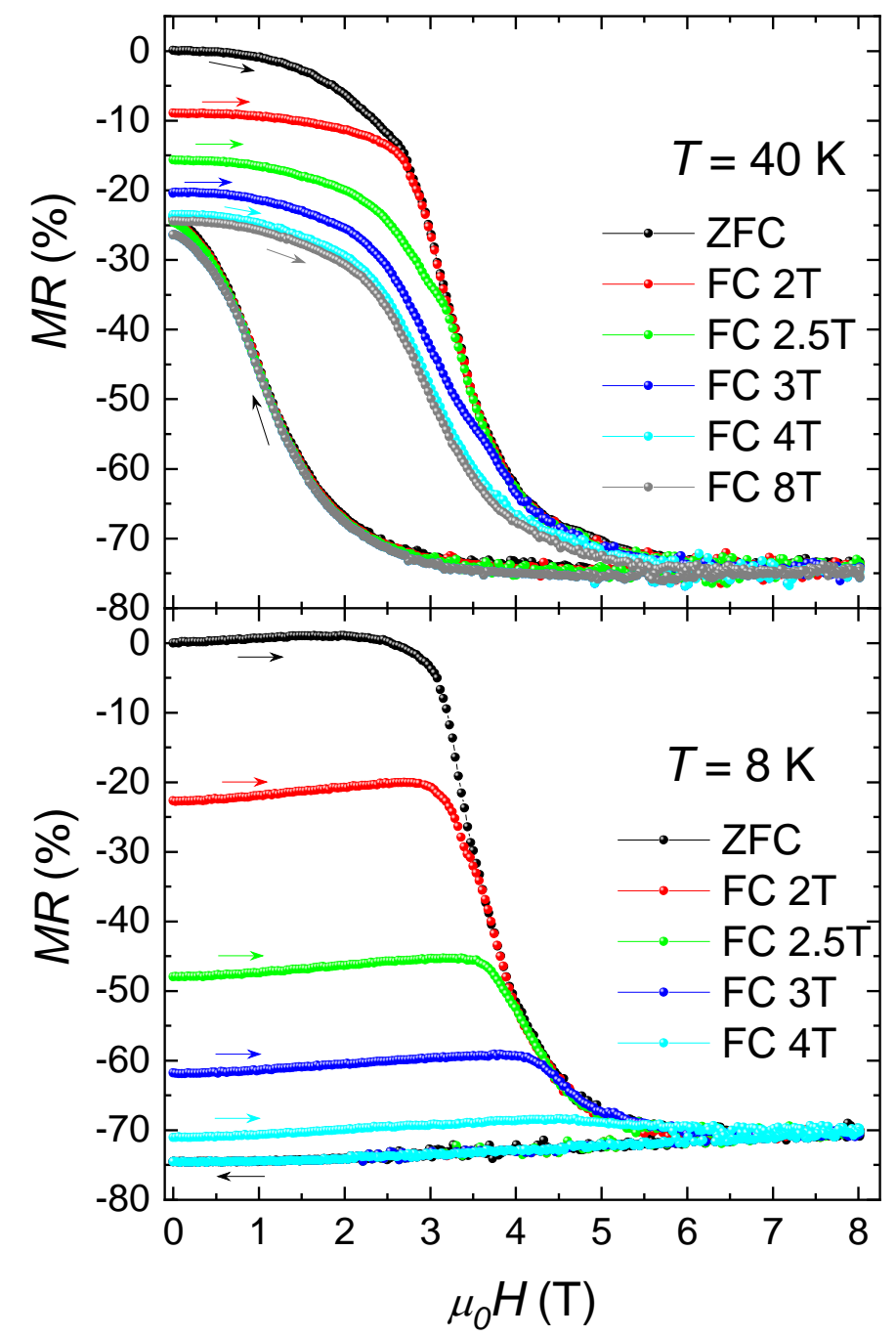

Figure 10. Isothermal magnetoresistance of $\mathrm{La}_{0.9} \mathrm{Ce}_{0.1} \mathrm{Fe}_{12} \mathrm{~B}_{6}$ at some selected temperatures of $8 \mathrm{~K}$ (lower panel) and $40 \mathrm{~K}$ (upper panel) taken after cooling the sample in different magnetic fields.

\section{CONCLUSION}

In summary, we have performed a systematic study of the magnetic and transport properties of $\mathrm{La}_{0.9} \mathrm{Ce}_{0.1} \mathrm{Fe}_{12} \mathrm{~B}_{6}$ as a function of temperature and magnetic field. The itinerantelectron metamagnet exhibits many anomalous phenomena in the magnetization and electrical resistivity results including large thermal hysteresis, multistep staircase-like metamagnetic phase transitions below $8 \mathrm{~K}$ and a strong field-driven irreversibility. Giant negative magnetoresistance effects have been found across the first-order AFM-FM and PM-FM metamagnetic transitions which are accompanied by huge magnetic field hysteresis. The magnetoresistance ratio associated with the field-induced AFM-FM transformation in $\mathrm{La} 0.9 \mathrm{Ce}_{0.1} \mathrm{Fe}_{12} \mathrm{~B}_{6}$ compound is as large as $\mathrm{MR}=-77 \%$ at $25 \mathrm{~K}$. 


\section{Data Availability Statement}

The datasets generated during and/or analysed during the current study are available from the corresponding author on reasonable request.

\section{References}

[1] A. Fujita, S. Fujieda, Y. Hasegawa, and K. Fukamichi, Phys. Rev. B 67, 104416 (2003).

[2] H. Yamada, and T. Goto Phys. Rev. B 68, 184417 (2003).

[3] D.P. Kozlenko, E. Burzo, P. Vlaic, S.E. Kichanov, A.V. Rutkauskas, and B.N. Savenko, Sci. Rep. 5, 8620 (2015).

[4] N.H. Duc, D.T. Kim Anh, and P.E. Brommer, Physica B 319, 1 (2002).

[5] L.V.B. Diop, O. Isnard, and J. Rodríguez-Carvajal, Phys. Rev. B 93, 014440 (2016).

[6] S. Fujieda, K. Fukamichi, and S. Suzuki, J. Magn. Magn. Mater. 421, 403 (2017).

[7] L.V.B. Diop, and O. Isnard, Appl. Phys. Lett. 108, 132401 (2016).

[8] L.V.B. Diop, and O. Isnard, Phys. Rev. B 97, 014436 (2018).

[9] L.V.B. Diop, and O. Isnard, J. Appl. Phys. 119, 213904 (2016).

[10] L.V.B. Diop, O. Isnard, Z. Arnold, J.P. Itié, J. Kastil, and J. Kamarad, Solid State Comm. 252, 29 (2017).

[11] G.I. Miletic, and Z. Blazina, J. Magn. Magn. Mater. 323, 2340 (2011).

[12] G.I. Miletic, and Z. Blazina, J. Alloys Compd. 430, 9 (2007).

[13] M. Rosenberg, T. Sinnemann, M. Mittag, and K.H.J. Buschow, J. Alloys Compd. 182, 145 (1992).

[14] Q.A. Li, C.H. de Groot, F.R. de Boer, and K.H.J. Buschow, J. Alloys Compd. 256, 82 (1997).

[15] M. Mittag, M. Rosenberg, and K.H.J. Buschow, J. Magn. Magn. Mater. 82, 109 (1989).

[16] K. H. J. Buschow, D. B. de Mooij, and H.M. van Noort, J. Less-Common Met. 125, 135 (1986).

[17] K. Niihara, and S. Yajima, Chem. Lett. 1, 875 (1972).

[18] Yu. B.Kuz'ma, G.V. Chernyak, and N.F. Chaban, Dopov. Akad. Nauk. Ukr. RSR Ser. A 12, 80 (1981).

[19] W. Jung, and D. Quentmeier, Z. Kristallogr. 151, 121 (1980).

[20] F. Mesquita, S. G. Magalhaes, P. Pureur, L.V.B. Diop, and O. Isnard, Phys. Rev. B 101, 224414 (2020).

[21] A. Barlet, J. C. Genna, and P. Lethuillier, Cryogenic 31, 801 (1991). 